\title{
A Wireless Multisensor Node for Long-Term Environmental Parameters Monitoring
}

\author{
Deguang Li (D), ${ }^{1}$ Tianhao Wu, ${ }^{1}$ Xiaohui Li, ${ }^{1}$ Qiurui He, ${ }^{1}$ and Zhanyou Cui ${ }^{2}$ \\ ${ }^{1}$ School of Information Technology, Luoyang Normal University, Luoyang 471934, China \\ ${ }^{2}$ College of Mechanical and Electrical Engineering, Zhengzhou Institute of Industrial Technology, Zhengzhou 451150, China
}

Correspondence should be addressed to Deguang Li; lideguang.00@163.com

Received 18 September 2020; Revised 6 December 2020; Accepted 15 December 2020; Published 28 December 2020

Academic Editor: Yang Li

Copyright (c) 2020 Deguang Li et al. This is an open access article distributed under the Creative Commons Attribution License, which permits unrestricted use, distribution, and reproduction in any medium, provided the original work is properly cited.

Environmental quality is a great concern to everyone, in order to realize the collection, upload, management, and visualization of parameters of atmospheric environment in real time. We propose a cheap, low-power, and fast deployment wireless sensor node for environmental monitoring, consisting of STM32 MCU, ESP8266, light sensor, rain sensor, UV sensor, seven-in-one sensor (including temperature, humidity, PM2.5, $\mathrm{PM} 10, \mathrm{CO}_{2}$, formaldehyde, and TVOC), and solar automatic tracking module. A customized $\mu \mathrm{C} / \mathrm{OS}$-III runs on the node, which controls the transmission of environment parameters collected by each sensor to the cloud server through the wireless network, and then the server receives, stores, and visualizes the data. In actual test, the node collects data once an hour, and the running power of the node is low and stable. Experimental results show that the node could achieve accurate collection and transmission and display the environmental data, and solar automatic tracking module could meet long-term running of the node in the night and continuous rainy days.

\section{Introduction}

With the development of human society, environmental pollution has become the first problem to be solved in the process of human development. Many researches have shown that many human diseases are caused by environmental problems; for example, aerobic exercise under air pollution could increase the risk of nose, pharynx, trachea, bronchus, and lungs, and ultraviolet radiation could cause human skin aging [1]. National Aeronautics and Space Administration (NASA) [2] points out that glaciers have shrunk, ice on rivers and lakes is melting ahead of time, plant and animal habitats have changed, and trees bloom ahead of expected dates due to global climate change. The main impact on climate change is mainly caused by $\mathrm{CO}_{2}$ emissions according to the European Commission [3]. Kench et al. [4] found that if the temperature exceeds 32.2 centigrade, the infant birth rate increases by $5 \%$, the length of the gestation period is reduced by an average of 6.1 days, and some babies are even born two weeks earlier. If climate change is ignored, global per capita (GDP) will be affected by climate and will fall by $7.22 \%$, and it will affect human intelligence by 2100 . As environmental quality is directly related to human sustainable development, real-time atmospheric environment monitoring is the top priority of environmental governance.

At present, environmental monitoring has been widely concerned. According to the monitoring scenarios, it can be divided into urban environmental monitoring and outdoor environmental monitoring. Urban environmental monitoring [5] mainly consists of the following subgroups: air pollution [6], light pollution monitoring [6], structural health [7], noise monitoring [8], and waste management [9]. The scenarios of outdoor environmental monitoring are always large-scale, long-distance, typical application scenarios that include intelligent agriculture [10-12], water quality monitoring [13], forest fire [14], etc. No matter urban environmental monitoring or outdoor environmental monitoring, some key environmental parameters should be monitored. Among them, the TVOC organic compounds in the atmospheric environment are dangerous to the human central nervous system and respiratory system, urban noise 
also causes specific damage to the auditory system, and urban light pollution and heat island effect also have some impact on human beings. Thus, in this paper, environmental parameters such as TVOC, noise, $\mathrm{CO}_{2}$, formaldehyde, UV intensity, light intensity, PM2.5, PM10, temperature, and humidity are monitored by the wireless sensor node.

Traditional fixed environmental monitoring stations are expensive in construction and management, and most of the data are recorded and entered manually, which is cumbersome and error-prone. Wireless sensor network (WSN) is an emerging key technology for future environmental monitoring, which are a low-cost, fast deployment, low maintenance and high quality service. WSN is usually made up of thousands of smart sensor nodes which monitor physical or environmental conditions; the specification and design of the platform and of all its components require cheap, scalable, and intelligent properties. The use of wireless sensor nodes had been proven to be faster in data collecting, less labor-intensive, and more cost-effective [14]. In order to conveniently and accurately monitor the atmospheric environment data, it is necessary to develop small-scale automatic environmental monitoring sensor nodes, which require miniaturization, intelligence, and scalability and can automatically connect to the Internet, automatically collect data, and send the data to the host server in intervals, so as to achieve stability and low-cost running, and can be easily deployed in batches.

The node proposed in this paper is employed with customized $\mu \mathrm{C} / \mathrm{OS}$-III to improve the operation efficiency and real-time data transmission. The main control chip adopts ARM STM32 chip with good expandability, which greatly improves the scalability and stability of the system. In order to meet the continuous demand of power supply, STC89C52RC is used to develop a solar energy automatic tracking module to collect solar energy and convert it into electrical energy, so as to ensure the power demand of the whole system.

\section{Related Work}

Wireless sensor network (WSN) is now widely used in environment monitoring $[15,16]$, which is always made up of thousands of smart sensor nodes that monitor physical or environmental conditions, such as water quality of river, soil moisture in precision agriculture, air quality, and other applications in different area. While all the application requirements of low cost, high number of sensors, fast deployment, long lifetime, low maintenance, and high quality of service are considered in the specification and design of the platform and of all its components [17], in order to fill all these requirements, many researchers put forward different sensor node design, network networking, data transmission, and node data prepossessing and data display for different application scenarios.

Zhang et al. [11] presented an information monitoring node based on automatic tracking solar-powered panel for paddy field environment; the node consists of information collecting sensors, STM32 MCU, LCD, GPRS, automatic tracking solar-powered module, and monitoring interface.
Joris et al. [18] proposed an autonomous Sigfox wireless sensor node for environmental monitoring, which consists of ATMEGA328P, low-power wide-area network protocols communication protocol, and a range of sensors; the characteristic of the node design is that the communication protocol has been optimized for low power consumption and offers competitive subscription prices. Maitra et al. [19] proposed a platform which was consisted by power source, sensors, microcontroller, wireless transceiver, gateway device, and cloud services, the platform that can be easily modified depending on the application and can be rapidly deployed; the main design idea enables a wide range of application specific layers to interact with other sublayers of the whole structure seamlessly, which is similar to many researches. Among the nodes listed above [11, 17, 18], the master control chip is ARM. Patil [17] also noticed that ARM microcontroller supports different features and algorithms for the development of industrial automation systems; many types of sensors using different wired or wireless technology were easily connected to the controller; also the chip is cheap and we can monitor and control the wireless sensor network remotely using Internet and web server. Also in many other scenarios, the master control chip is also based on ARM platform; thus, in this paper, we also use the chip based on ARM as the master control chip in the node.

While the wireless network used in [11] is based on GPRS, although the speed of GPRS is fast and supports long transmission distance and simple networking, however, due to the limitation of the station coverage, the signal is relatively weak in poor communication conditions and there is a communication dead angle. Thus, in order to overcome these limitations, various network communication technologies are deployed for different scenarios. Low-power wide-area network (LPWAN) protocols, such as LoRa (Semtech) [12], Sigfox (Sigfox Company) [13], or NBIoT (3GPP), have been developed; these protocols address the need for a long range communication system with limited data to transmit and exhibit increased energy efficiency. Also, some wireless node was based on RFID in [20, 21]. Mois et al. [22] presented three different IoT-based wireless sensors for environmental monitoring: one employing User Datagram Protocol based Wi-Fi communication, one communicating through Wi-Fi and Hypertext Transfer Protocol (HTTP), and a third one using Bluetooth Smart; all these three systems were implemented and proved the feasibility, energy autonomy, usability, and Internet connectivity facility; the experiment result revealed that they can be good candidates for IoT-based solutions. Al Azam et al. [23] also used Wi-Fi to monitor and control the environmental; unlimited control could be exercised by electrical parameters of an interconnected network operations.

Behera et al. [24] noticed that energy is a major constraint in the design of a WSN and proposed an improvement of the existing stable election protocol that implements a threshold-based cluster head selection for a heterogeneous network. Automatic tracking solar-powered module is used for consistent power supply in many researches for longterm running, which is very important for the node in WSN; thus in this paper, we also make use of automatic tracking 
solar-powered module for the node. Many other researches proposed different methods for efficiency routing and data secure such as Ahmad et al. [25] who presented the design and test of IEEE 802.15.4-compliant smart IoT nodes with multi-hop routing. Wu et al. [26] proposed a novel framework with dedicated combination of data prediction, compression, and recovery to simultaneously achieve accuracy and efficiency of the data processing in clustered WSNs. Bezzateev et al. [27] designed a system which allows protecting a sensor network from unauthorized topology changes keeping the property of scalability and security, which enables efficient and fast network initialization, received information processing, and handling potential topology changes.

Based on previous works, we propose a low-cost, lowpower, and fast deployment micro wireless sensor environment test node, which uses STM32 as the main control chip, ESP8266 module for Wi-Fi communication, light intensity sensor, raindrop sensor, UV intensity sensor, seven-in-one sensor, and solar automatic tracking module, and the node is equipped with a customized $\mu \mathrm{C} / \mathrm{OS}$-III for control and management.

\section{Overview of System Design}

The system mainly consists of five parts, which are sensor node, gateway, solar power system, database server, and web server; the overall framework of the system is shown in Figure 1.

Sensor node monitors real-time environmental data information and sends the collected data to the nearby gateway through Wi-Fi. Gateway collects the received data and sends it to the database server through the external network. Database server receives the data and maintains it, and the web server visualizes the data for human interaction. In order to ensure power demand of the system and continuous power operation under the condition of poor illumination, each sensor node has a full range of solar light tracking system, which can collect solar energy and store it for continuous power supply. Data acquisition, wireless transmission, data storage, and data visualization are implemented based on the hardware.

\subsection{Hardware Design}

3.1.1. The Master Control Chip. The main control chip used in the node is STM32F103ZET6, which is based on ARM Cortex-M core, and has the characteristics of high performance, low cost, small size, low power consumption, compact code density, and simplified instruction set. The embedded processor is the core part in our design. And an optimized operating system $\mu \mathrm{C} / \mathrm{OS}$-III runs in the processor to drive and control the equipment and complete the collection of atmospheric environmental parameters. Also, data processing program is embedded for flexible processing, safe storage, and other functions. The chip has rich I/O interfaces, 3 12-bit AD converters, 2 12-bit DA converters, and 12 DMA channels and supports peripheral timing controller, ADC, DAC, I2S, SPI, I2C, and USART, which has good scalability and stability, enough to meet the requirements of system deployment and operation, and the physical hardware connection of the node is shown in Figure 2.

3.1.2. Sensors. The nodes in this paper include five sensors; the sensors are UV sensor, GT-302 light sensor, raindrop sensor, noise sensor, and SM300D2 seven-in-one sensor (including temperature, humidity, PM2.5, PM10, formaldehyde concentration, $\mathrm{CO}_{2}$ concentration, and TVOC concentration). The node collects eleven types of environmental data, and some parameters of each sensor are shown in Table 1.

The seven-in-one sensor can simultaneously monitor seven kinds of data information such as $\mathrm{CO}_{2}$, formaldehyde concentration, PM2.5, TVOC concentration, PM10, temperature, and humidity and output data through the serial port. In addition to the three datasets of $\mathrm{CO}_{2}$ concentration, formaldehyde concentration, TVOC concentration, it needs to be warmed up for about two minutes after powering on, and the other data can be displayed immediately after powering on. The collected data can be output through the digital serial port; also, they can be output through the RS485 data bus and UART serial port. We use the UART output mode in this paper, so the sensor pins are connected to the microprocessor's PB10 and PB11, where PB10 is the USART3_TX of the microprocessor and PB11 is the USART3_RX of the microprocessor.

The model of ambient light intensity sensor is GY-302; we can directly get digital output by ignoring the conversion of complex electrical signals to light intensity, and its sensitivity is close to the spectral characteristics of visual sensitivity. The GY-302 light sensor uses I2C bus to receive and transmit data, which is connected to PB3 and PA15 ports of microprocessor, respectively, and writes instructions to the sensor through I2C bus to complete one single reading of light intensity data of the environment, and then the data are transmitted to the microprocessor through I2C bus.

The UV sensor used in this article is specially designed for the occasions that require high reliability and accuracy to measure the ultraviolet index (UVI) and is suitable to measure the total UV intensity of sunlight. The total intensity value can be compared with the World Health Organization ultraviolet index classification standard. This sensor can detect the ultraviolet wavelength of $200-370 \mathrm{~nm}$, which has the characteristics of fast response speed and full interchange ability.

Both UV sensor and noise sensor output analog signals. UV sensor is connected to the PA6 port of the microprocessor and noise sensor is connected to the PA7 port of the microprocessor. The two sensors share the ADC1 digital to analog converter of microprocessor and convert the electrical signal into 12-bit digital signal through the built-in ADC sensor.

The output data of rain sensor used in this paper have the advantages of clean signal, good waveform, and strong driving ability, which outputs data through the TTL level and outputs a low level when raindrops are detected and a high level when there are no raindrops, which is connected to the PD9 port of the microprocessor. 


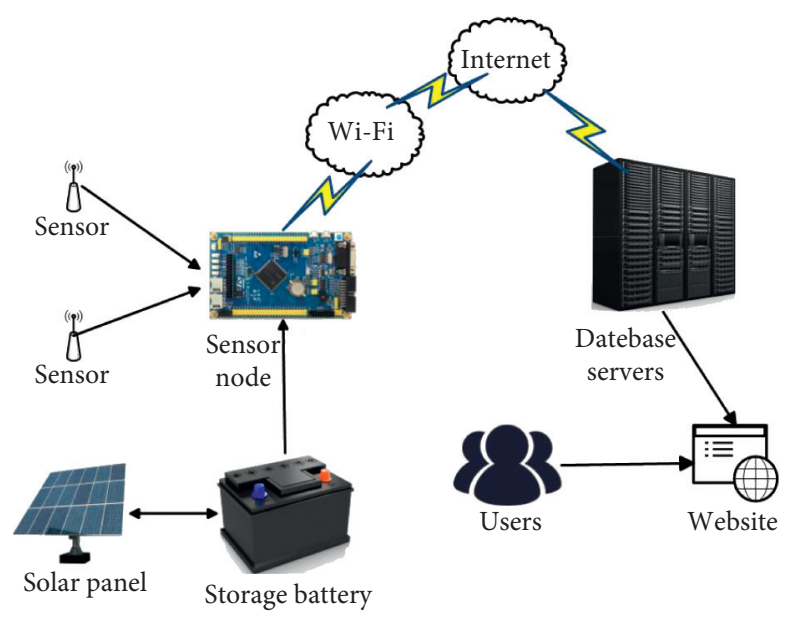

Figure 1: Overview of the system design.

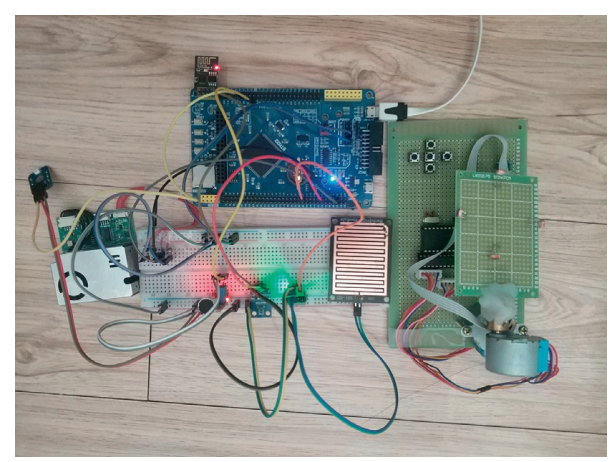

FIgURE 2: Hardware connection of the node.

TABLE 1: Detail parameters of each sensor.

\begin{tabular}{|c|c|c|c|c|c|}
\hline Sensor & Parameter & Voltage (V) & Range & Precision & Transmission \\
\hline GY-302 & Light intensity & $3.3-5$ & $0 \sim 65535 \mathrm{~lx}$ & $11 \mathrm{x}$ & $\mathrm{I} 2 \mathrm{C}$ \\
\hline UV & UV intensity & $3.3-5$ & 0-12UV & $\pm 1 \mathrm{UV}$ & $\mathrm{ADC} 1$ \\
\hline RAIN & Rain or not & $3.3-5$ & Rain/no rain & & USB \\
\hline \multirow[t]{4}{*}{ VOICE } & Noise intensity & $3.3-5$ & $20 \sim 150 \mathrm{~dB}$ & $\pm 1 \mathrm{~dB}$ & $\mathrm{ADC1}$ \\
\hline & $\mathrm{eCO}_{2}$ & 5 & $400 \mathrm{ppm} \sim 5000 \mathrm{ppm}$ & $\pm 100 \mathrm{ppm}$ & \\
\hline & $\mathrm{eCH}_{2} \mathrm{O}$ & 5 & $1 \mu \mathrm{g} \sim 1000 \mu \mathrm{g}$ & & \\
\hline & TVOC & 5 & $0 \mu \mathrm{g} \sim 2000 \mu \mathrm{g}$ & & \\
\hline \multirow[t]{4}{*}{ SM300D2 } & PM2.5 & 5 & $5 \mu \mathrm{g} \sim 1000 \mu \mathrm{g}$ & $\pm 10 \%$ & USART3 \\
\hline & PM10 & 5 & $5 \mu \mathrm{g} \sim 1000 \mu \mathrm{g}$ & $\pm 10 \%$ & \\
\hline & Temperature & 5 & $-40^{\circ} \mathrm{C} \sim 125^{\circ} \mathrm{C}$ & $\pm 0.5^{\circ} \mathrm{C}$ & \\
\hline & Humidity & 5 & $0 \sim 100 \%$ & $\pm 3 \% \mathrm{RH}$ & \\
\hline
\end{tabular}

3.1.3. Wi-Fi Module. As the traffic and bandwidth required by each sensor node are very small, in order to reduce the communication cost, all nodes in this paper are not equipped with separate GPRS network. Instead, all sensor nodes in a certain area are connected to a $\mathrm{Wi}-\mathrm{Fi}$ gateway, which is configured in a certain area. The gateway receives data from sensor nodes in a certain range and then uploads the data to the server for permanent storage. Deploying multiple sensors in one area to collect data at the same time can ensure the accuracy and fault tolerance of the data. If a sensor fails in this area, other nodes can still collect data normally, which helps improve the overall robustness of the system.

The network topology of the node is shown in Figure 3. We use Wi-Fi wireless network communication to achieve data transmission between the node and the gateway. The Wi-Fi network has a wide coverage area and can cover 100 meters without obstructions. It has high reliability and no wiring, is safe for humans, and has a small impact on the environment. It can reduce the impact on the environment when it is deployed in the field, so using the Wi-Fi protocol to connect sensors and gateways is a good choice. 


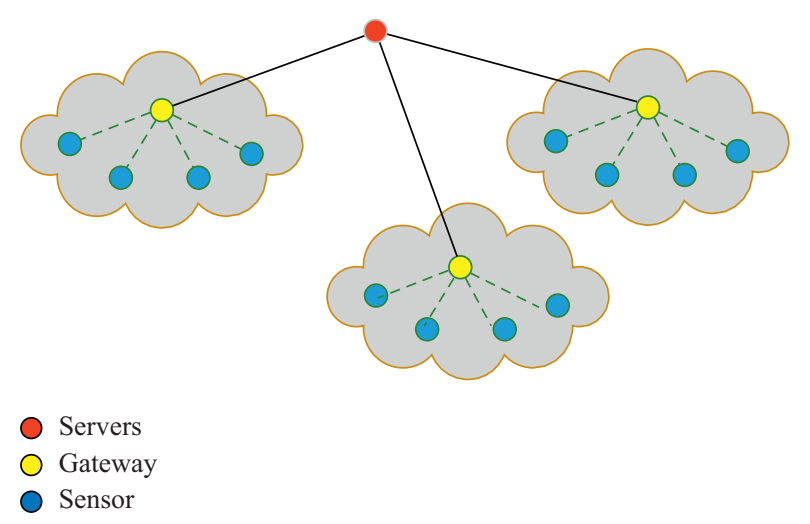

FIGURE 3: Network topology of sensor nodes.

Based on the above considerations, we use ESP8266 wireless communication module to realize the communication function.

In this design, the module sends AT command through the serial port to initialize the esp8266 module after the node is powered on each time. Then, the sensor packs and sends the data after receiving the data. At the same time, the ESP8266 module can work in the mode of AP and STA; at this time, the node can be used not only as the STA of wireless $\mathrm{Wi}-\mathrm{Fi}$, but also as the AP of other sensor nodes to provide data forwarding services. The gateway acts as the AP access point of the sensor nodes, and all sensor nodes are connected to the gateway through the ESP8266 module. The gateway and the sensor nodes are connected via Wi-Fi, communicate through the TCP/IP protocol, and then perform data transmission in the $2.4 \mathrm{GHz}$ frequency band.

3.1.4. Solar Automatic Tracking Module. This module is an important module to ensure the long-term endurance of the system. In this module, STC89C52RC chip with low energy consumption and four photosensitive resistors are used. The voltage of each photosensitive sensor is read through PCF8591chip to get the angle difference between the direct sunlight angle and the current solar panel. Then, the solar panel is moved to an angle perpendicular to the light by using ULN2003 to drive the stepping motor. This module is designed to maximize the efficiency of solar energy conversion. Compared with the traditional fixed solar system, this module can make the most reasonable dynamic adjustment according to the direct angle of the sun in different seasons and times.

The realization of this design is to place the four light sensors on the four edges of the solar panel outward 45 degrees, and the position of the photosensitive resistor is shown in Figure 4. Through the ADC module, the voltage of the four photosensitive resistors is converted in real time to determine the deviation of the current sun and then drive the stepping motor to make adjustment. Compared with the traditional time-based automatic adjustment method, this method has higher accuracy and simple algorithm, which can be realized without complex design.

\subsection{Software Design}

3.2.1. Software Architecture. The software flowchart of the node is shown in Figure 5. First, after powering up the node, operating system and individual sensor hardware of the node are initialized, and then two main processes are created, which are the sensor working process and the $\mathrm{Wi}-\mathrm{Fi}$ working process. Detailed steps of these processes are presented as follows. Firstly, the $\mu \mathrm{C} / \mathrm{OS}$-III is initialized; task stack size, task priority, task control block naming, task stack setting, and startup function parameters required by all processes are defined. Three process control blocks are created, which are Start_Task_TCB (operating system process), Item_Task_TCB (sensor process), and WIFI_Task_TCB (Wi-Fi process). Then, all hardware and sensors are initialized by the main function, next, all the parameters required by the operating system process are initialized, and OSTaskCreate() function is created, which creates the Start_Task_TCB process to run the UC/os-iii operating system. Finally, the sensor work process Item_Task_TCB and WiFi process WIFI_Task_TCB are continued, created by TaskCreate() function. In the Item_Task_TCB process, five functions named STOO_Get_Data(), UV_Read_Data(), VOICE_Read_Data(), RAIN_Read_Data(), and Get_Sunlight_Value() are periodically called to get environment data from the five different sensors separately; all the data are packed and sent by the process of WIFI_Task_TCB.

When the program of the sensor node works correctly, each function is ready to collect data through I2C bus, ADC module, etc. And then the data collected by the sensor are sorted and encapsulated and sent to the remote server by ESP8266 module. In this way, we can observe the changes of environmental data in real time.

3.2.2. Migration of Operating System. $\mu \mathrm{C} / \mathrm{OS}-\mathrm{III}$ is $\mathrm{mi}-$ grated to the sensor node, which is a priority-based multitasking kernel operating system that can be tailored, upgraded, solidified, and deprived; the kernel can be trimmed to a minimum size of $4 \mathrm{kB}$, which needs very small resources to run and has very high efficiency, and the tasks can be switched in real time. During the migration, the uC-CPU, uC-LIB, and UCOS-III in the project are mainly modified, and the UCOS_BSP and UCOS_CONFIG files are configured at the same time. Finally, a lighting function is created to verify the success of the system migration.

$\mu \mathrm{C} /$ OS-III operating system [28] has five task states by default, which are sleep state, ready state, run state, wait state, and interrupt service state. In this system, the $\mu \mathrm{C} / \mathrm{OS}$ III task is created by calling the OSTaskCreate() function, and the task code is stored in ROM, but ostaskcreate() function is required to inform the operating system about the task. After the task is completed, the task will be deleted by the OSTaskDel () function. In the single-CPU system of this article, the application needs to select the highest priority task from the ready queue of the $\mu \mathrm{C} / \mathrm{OS}$-III operating system by calling OSStart() or OSIntExit() or OS_TASK_SW(). 


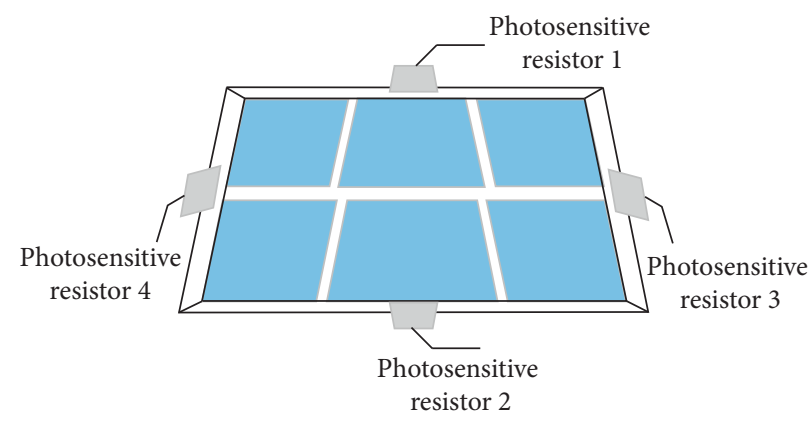

FIgure 4: Position of photosensitive resistor.

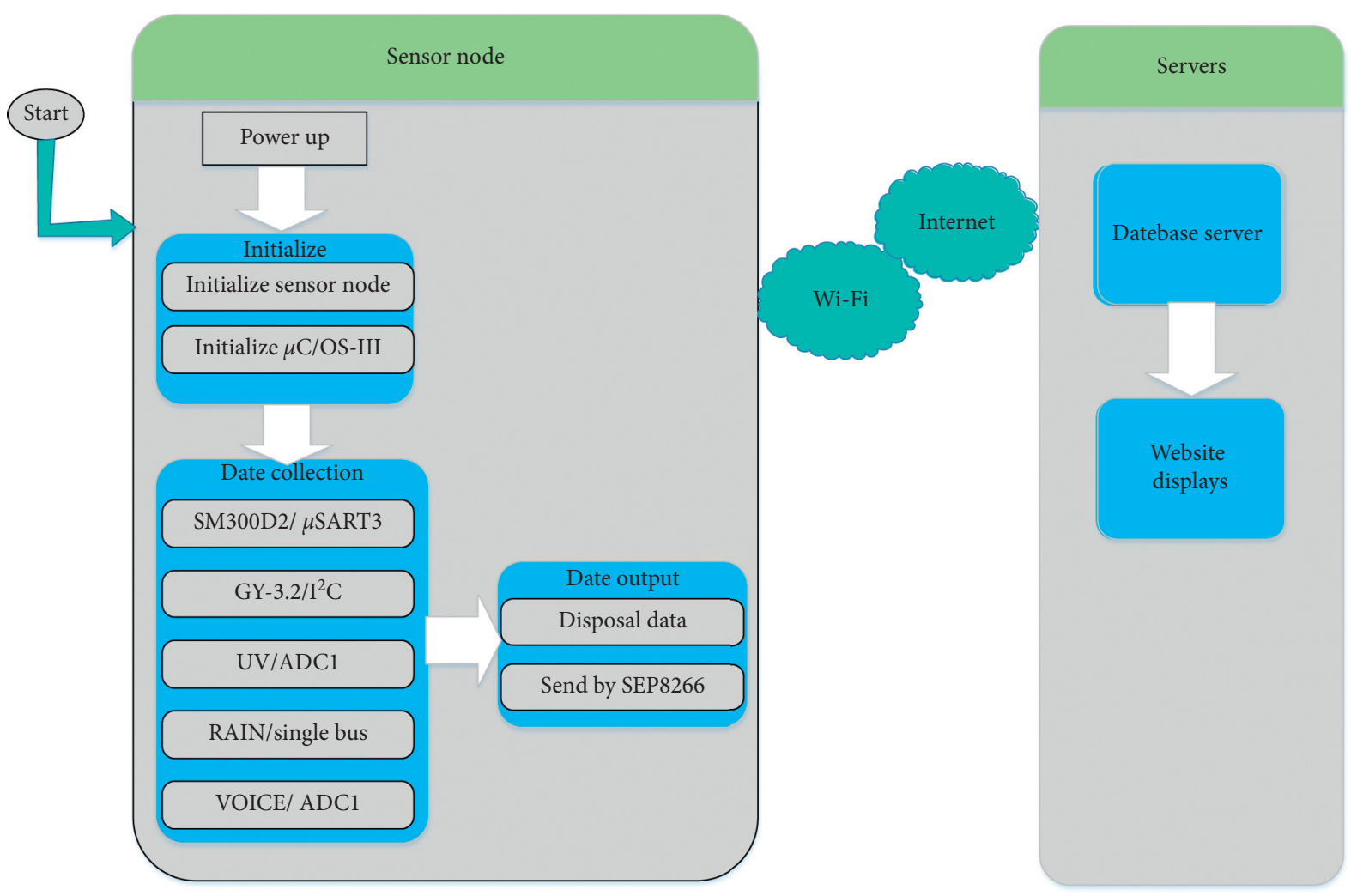

FIgURE 5: Software flowchart of the system.

3.2.3. Program of Each Sensor. As SM300D2 sensor outputs all data through UART serial port, this article uses the USART3 interrupt method of STM32 microcontroller to read data, and the baud rate is 9600 bps. The sensor pins are connected to PB10 and PB11 of the microprocessor, where PB10 is the USART3_TX of the microprocessor and PB11 is the USART3_RX of the microprocessor. Each time, it will send 17 groups of nine bits of data; each group contains eight data bits and one stop bit. Then, the microcontroller receives data through the interrupt mode of USART, retain eight data bits, and discard the ninth stop bit, until receiving 17 groups of data completely and calculating their checksums. Detailed steps are as follows: (1) calling the sensor interface function in the main program, waiting for the data to be finished. (2) Then, processing the raw data and generating meteorological data. (3) In the data returned by the sensor, the first set of data is a fixed sensor address and its address is $0 \times 3 c$, and the second set of data is the fixed version number of the sensor and its address is $0 \times 02$; therefore, as long as these two groups of fixed data are received, the later data is the environmental data collected by the sensor.

The ambient light intensity sensor uses I2C bus for data transmission, and certain steps should be followed for each data acquisition. In this paper, one measurement is adopted, that is, one acquisition and data transmission carried out after each power on; then, the chip automatically powers off; the steps are shown in Figure 6. The execution is first to execute the code to power on the components and write instructions to the sensor; after waiting for a certain time delay, the power supply is stable. Next, selecting the 


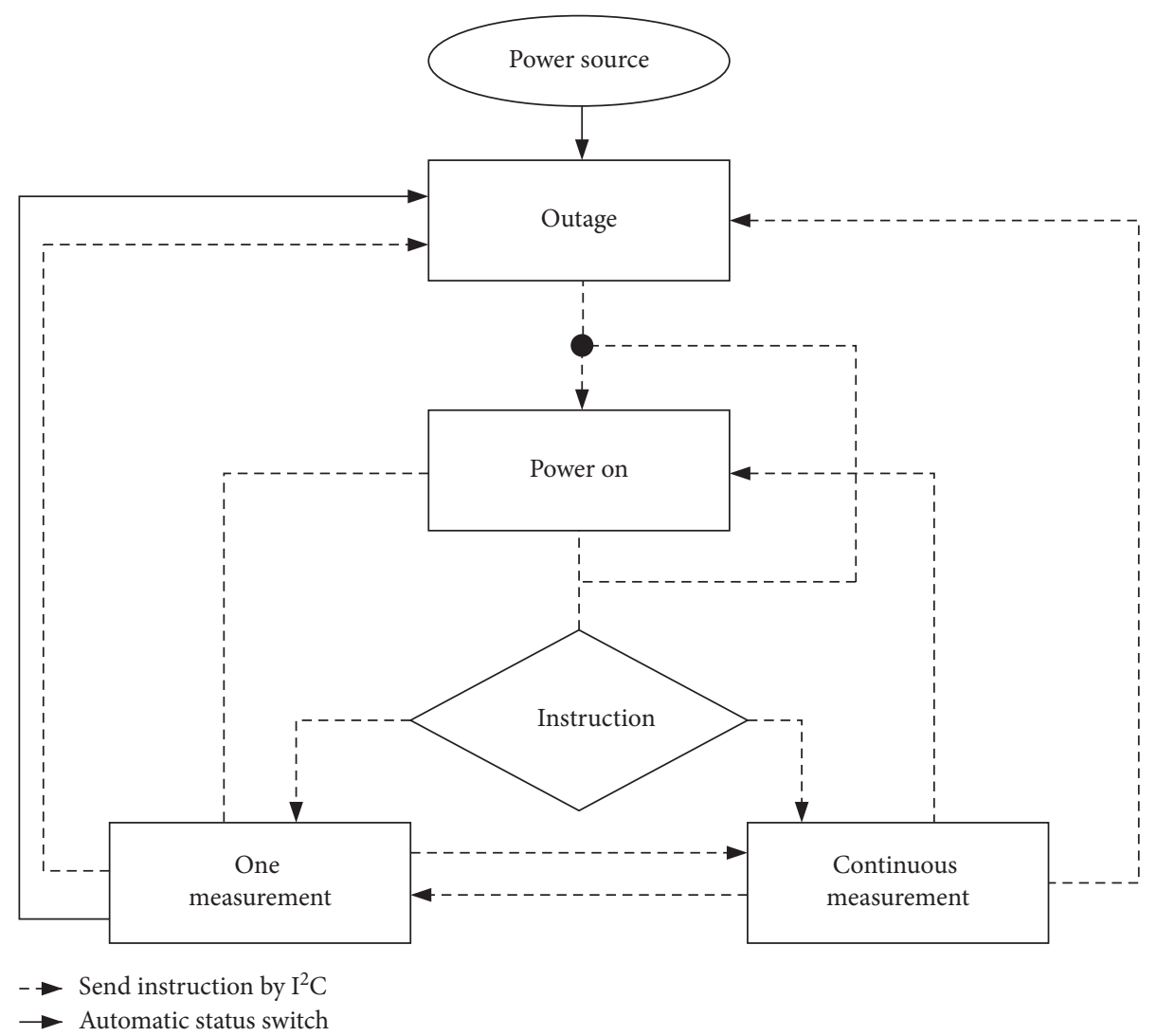

Figure 6: Procedure of light intensity sensor measurement.

acquisition precision with code, high-precision scheme is adopted in this paper and acquisition accuracy is 1LX, thus writing a high-accuracy acquisition instruction $0 \times 10$ (Single_Write_BH1750 $(0 \times 10))$ to the sensor, waiting for $180 \mathrm{~ms}$ and the sensor acquisition to finish; then, the data is read according to the $\mathrm{I} 2 \mathrm{C}$ communication signal. The chip then automatically powers off and waits for the next poweron execution.

The UV sensor is connected to the PA7 port of the microprocessor; this port is a channel for the ADC1 converter built into the microprocessor. The physical signal collected by the ambient ultraviolet intensity sensor needs signal conditioning to perform analog-to-digital conversion. In this paper, we use the ADC channel built in STM32 microprocessor to process the voltage analog signal; after conversion by ADC converter of microprocessor, the data will be compared with the UV intensity comparison table, and then the corresponding UV level will be returned. In order to ensure the accuracy of the calculation of the UV intensity, the average value of 10 conversions is calculated as the final value.

The rain sensor used in this article uses a wide-voltage LM393 comparator; thus, the output data has the advantages of clean signals, good waveforms, and strong driving capabilities. At the same time, it is equipped with a potentiometer to adjust the sensitivity according to demand. The digital switch output pin is connected to the PD9 pin of the microprocessor, and the microprocessor receives the relevant level signal of the pin through the interface function int8_t RAIN_Read_Bit(). The sensor board outputs high level when there are raindrops and low level when there is no water drop.

When the voice sensor is powered on, the chip starts to collect the signal and output the analog signal and automatically converts the voltage signal into the analog signal through the ADC channel of the single-chip microcomputer. Then, the function GetADCValue() is called through the interface of VOICE_Read_Data(); the function reads the ADC value 10 times in a row and then averages it to get a relatively accurate value; the corresponding voice value can be calculated by the voice formula (1):

$$
\text { voice }=\sum_{i=1}^{10} 10 * \log \frac{\text { value }}{0.001}
$$

3.2.4. Program of Solar Automatic Tracking Module. The default operation mode of solar module is manual mode after powering on; at this time, we can switch manual mode to automatic mode through mode switch button (key_state). In manual mode, stepper motor can be, respectively, controlled to rotate in different directions by four buttons, so as to achieve the corresponding up, down, left, and right rotation of the lighting panel. In this paper, we switch working mode to automatic mode after powering on. In automatic mode, the single-chip microcomputer detects the light intensity values of the four directions of the lighting board 
through four photosensitive resistors on the lighting board and then controls the stepper motor to make the stepper motor always rotate in the direction of strong light intensity. When the light intensity collected by the sensors in the up and down direction and in the left and right direction is the same, stop rotating, so as to ensure that the front of the lighting board moves perpendicular to the side with the largest light intensity value to receive solar energy to the maximum.

\section{Results and Discussion}

Due to the limitation of novel coronavirus disease prevention and control, our system could not be tested on a large scale. Validation tests were made in our community we live in, where sensor nodes were placed outdoors in a distance of 80 meters of the network gateway, and our computer received the collected data by the communication with the gateway. When the node is powered on, related processes are created automatically, and the software and hardware of the system are initialized. In order to test the stability of the system and the endurance of solar energy, we set the time interval for automatic collection of environmental data by the program to be every one minute. In the actual test, each data collection takes about two seconds to complete. When data collection of the node is completed, the sensor sends data to the nearby gateway. The gateway receives the data through the constantly scanned network port and intercepts the strings in the data to obtain the relevant sensor parameters and the unique identification number of each node. Finally, the gateway transmits the data to the database server for storage.

By practical test, we find the instantaneous core current of the STM32 system is about $0.06 \mathrm{~A}$ when the node is in the data acquisition state (all the sensors start to collect data), and the current of different sensors is about $0.08 \mathrm{~A}$; thus, the total power consumption of nodes in data acquisition is about $0.65 \mathrm{~W}$, the working current of $\mathrm{Wi}-\mathrm{Fi}$ transmitting data is about $0.1 \mathrm{~A}$, and thus power consumption of the node during data transmission is about $0.8 \mathrm{~W}$. When the node goes into sleep state, STM32 core is in power-on state, and other circuits are in standby state, the core current of the STM32 is about $0.05 \mathrm{~A}$, and the current of other standby circuits is about $0.01 \mathrm{~A}$; at this time, the power consumption of the node is about $0.3 \mathrm{~W}$. The node collects data once an hour, works for 1 minute every hour, and is on standby for 59 minutes; thus, the power consumption of the node in one day is about 7.4 Wh. As shown in Figure 7, the instantaneous power consumption of the system over a period of time is shown. In general, the overall power consumption of system nodes is significantly lower than that of other system nodes.

The environmental detection node in this paper is effective in sensing and collecting the environmental data in real time. The running power of the node is very low when it works. According to the actual measurement, the power of the whole system is only $0.35 \mathrm{~W}-0.7 \mathrm{~W}$, which can meet the needs of low-power continuous work. And the sun automatic tracking module is able to track the sun's position and provide continuous power energy. At the same time, the robustness and stability of the system are guaranteed. Since we have deployed three nodes in the same observation area at the same time, all three nodes collect data, and the gateway receives the data from three nodes and sends them to the database server. When the web server displays the environment data, it calculates the mean value of the same data collected by three nodes at the same time and displays the environment data in the form of mean value.

In order to display the collected environment data interactively, we designed different visualization schemes for different environment data. As shown in Figure 8, there are eleven kinds of real-time environmental data, which display the collected eleven environmental data in a unified and realtime way. At the same time, we also provide a specific visualization interface for each of the environmental data, which will not be displayed due to space constraints. Figure 6 is drawn by pyecharts, the third-party visualization library of Python. Twelve list parameters, including time, temperature, humanity, PM2.5, PM10, light, UV intensity, ech2o, $\mathrm{CO}_{2}$, TVOC, rain, and noise, are passed into the line() method of pyecharts library and are distinguished by multiple colors. The $x$-axis represents the time period, and the other eleven list parameters are the names of eleven environment data; the content in parentheses is the unit of measurement of the data. Due to the huge amount of data, it is inconvenient to directly display all the data. Thus, the datazoom_opts() method is re-inherited in the graph and a drag handle is added, so that you can directly drag on the graph to view the eleven types of environmental data corresponding to the moment.

In addition to drawing the real-time data graph, in order to understand the changes of some environmental data in a period of time, the graph of data changes in a certain period of visualization is added, and different visualization technologies are used to display. As shown in Figure 9, the changes of $\mathrm{CO}_{2}$, TVOC, and average temperature in the past ten days are shown. The column graph and graph chart are displayed at the same time; among them, $\mathrm{CO}_{2}$ and TVOC are displayed by column graph, and the corresponding ordinate parameters are on the left side of the graph, indicating the average $\mathrm{CO}_{2}$ and TVOC volume of the day. Average temperature is displayed with a line graph, and a signed scale is added on the right side of the graph. The right side of the $y$ axis is divided into adjacent scales with a difference of five, and corresponding temperature parameter is on the right side, indicating average temperature of the day. The average values of $\mathrm{CO}_{2}$, TVOC, and temperature in a day can be clearly observed through this figure, and the time cycle can be customized according to user's demand, such as one week or one month.

Figure 10 shows the PM2.5 and PM10 scatter graph in the past 13 days. The scatter size in the chart is drawn according to the size of the data value, which can clearly display the environmental data. At the same time, different colors are used to distinguish the data value beyond the range. Through the graph, the changes of the environmental data in different time periods are clearly displayed, and the time cycle of the chart can be based on specific requirement, such as one week or one month. The $x$-axis represents the 


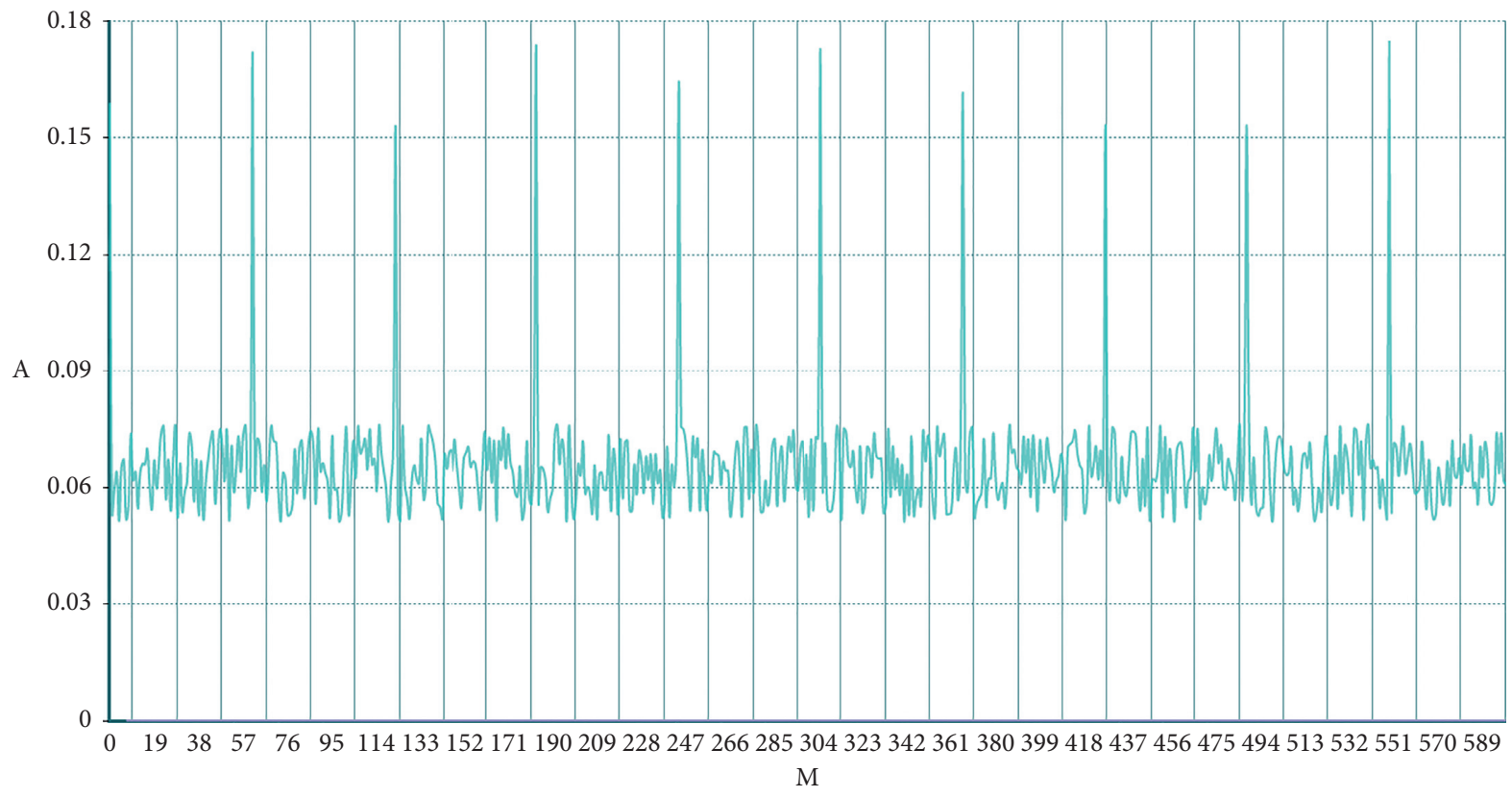

FIgURE 7: Instantaneous power consumption of the system.

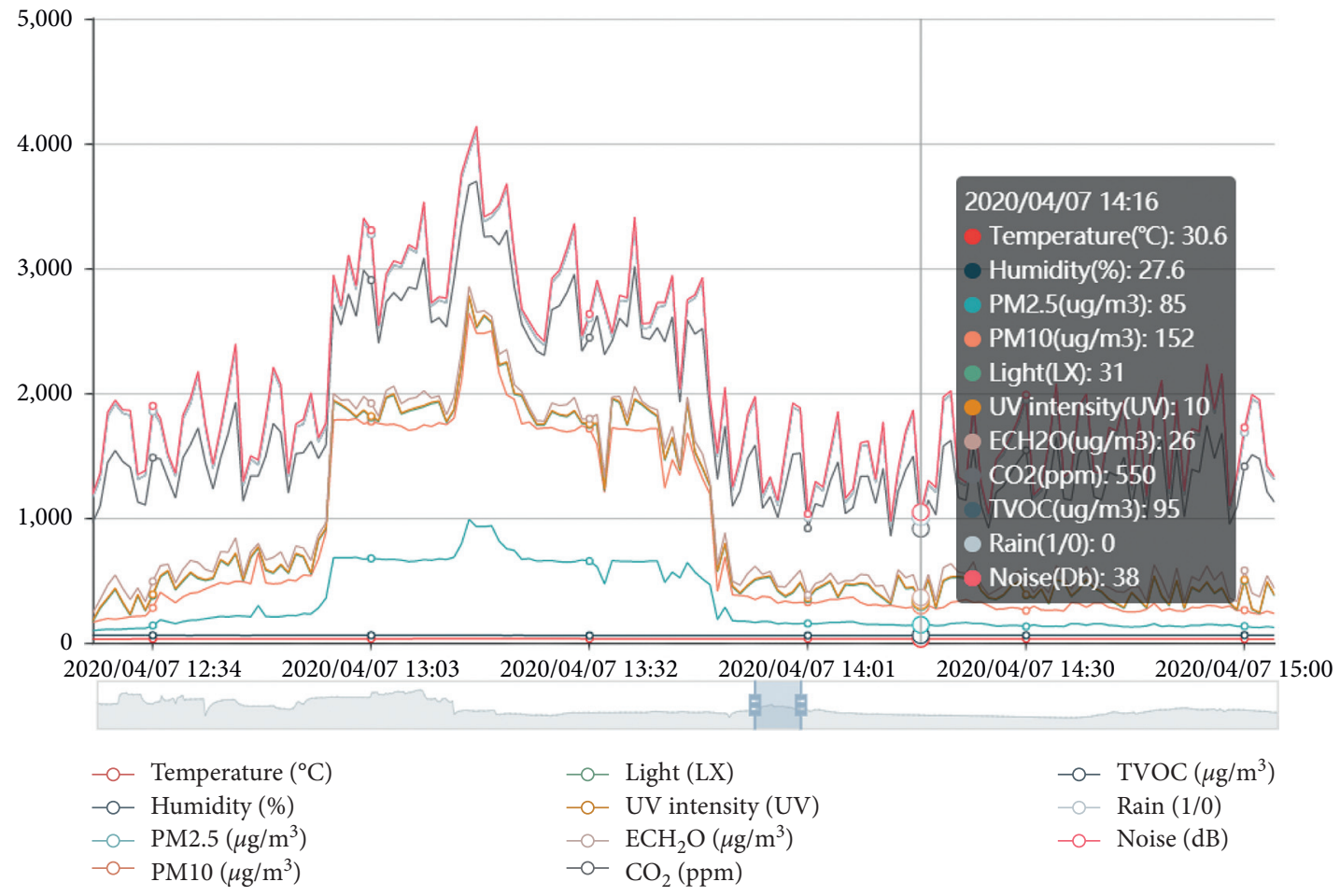

Figure 8: Comprehensive diagram of real-time data of eleven kinds of environments.

time, and the $y$-axis represents the average value of PM2.5 and PM10 in one day. When extracting data from the database, the average value of PM2.5 and PM10 in a day is calculated, and then the average value is inserted into the value array. According to the array value, two kinds of color scatter are used to distinguish the scatter. The size of scatter point is determined by the data value; users can more intuitively feel the difference of data. We also add a visual mapping of inverted triangle at the bottom corner, and users can click different data points on the vertical axis of inverted triangle with a mouse, which can make the small balls in the pattern similar to the value flash, which is 


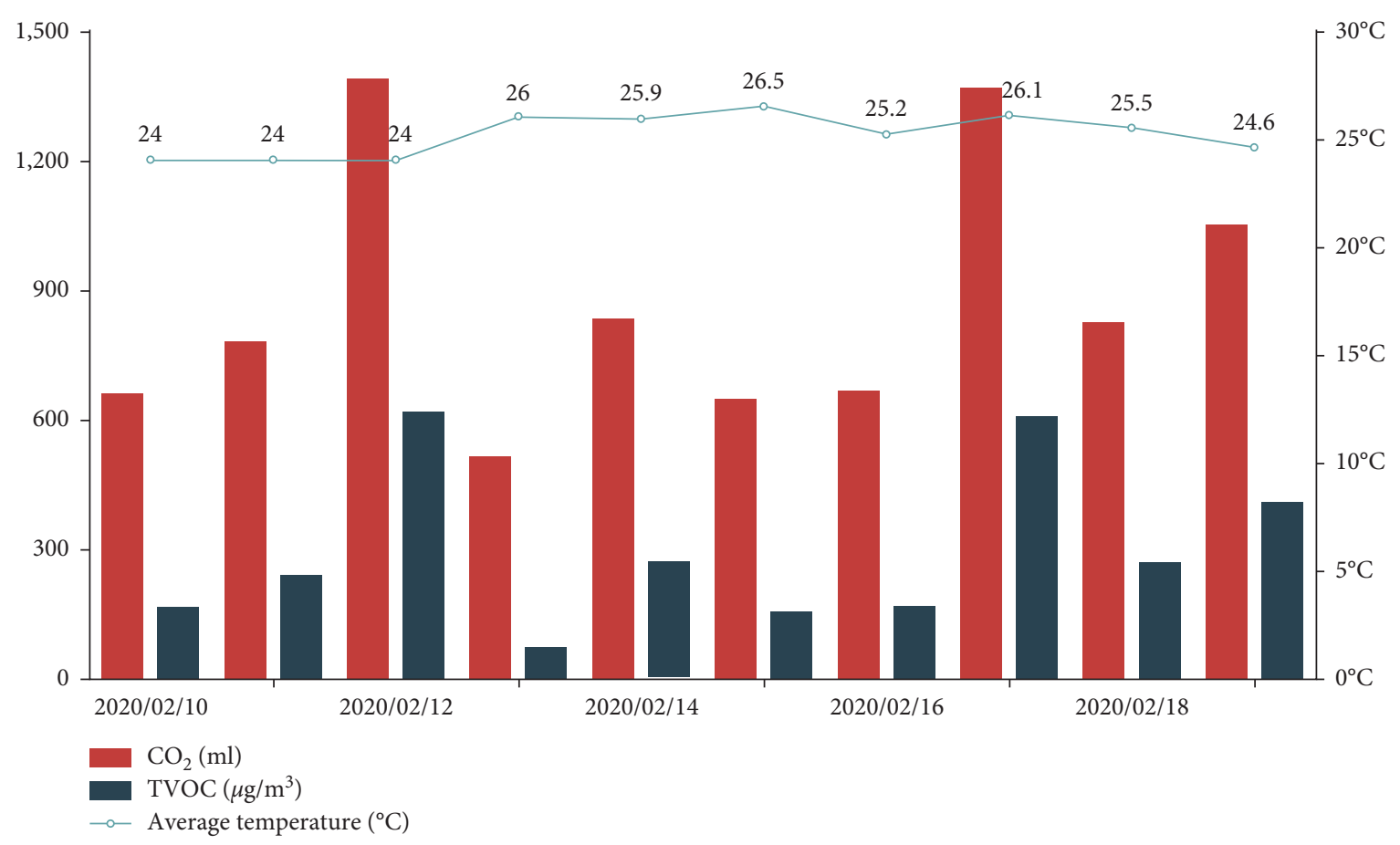

Figure 9: Trend of average temperature, $\mathrm{CO}_{2}$, and TVOC in a period.

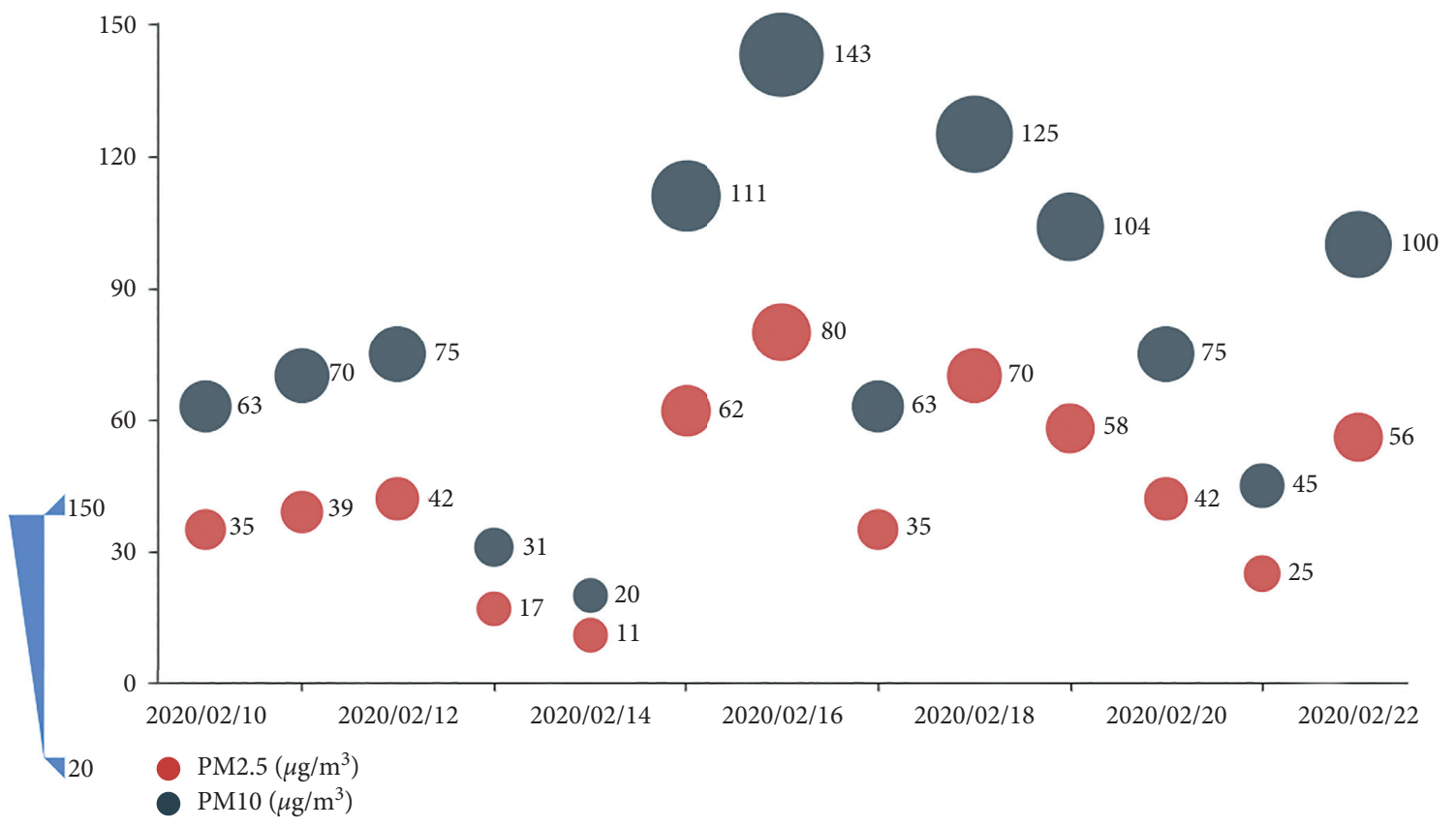

FIgURE 10: Data changes of PM2.5 and PM10 in a period.

convenient for quickly finding the location of the scattered points.

\section{Conclusions}

This paper designs and implements a cheap, low-power, and rapid-deployment wireless sensor environment monitoring node and realizes the real-time collection of a variety of environmental data. The data transmission between the node and the gateway is realized by using the $\mathrm{Wi}$-Fi wireless network. Multiple sensors are deployed in a region to collect data at the same time, which can ensure the accuracy and fault tolerance of the data. The power of the node is low in the working and running state, which can meet the low 
power consumption and continuous working demand of the node. At the same time, the node is equipped with the solar automatic tracking module, which can track the orientation of the sun in real time, continue the production and storage of power energy, and meet the long-term working demand of the node in various weather environments. Finally, through experimental verification, the node achieves the goal of real-time collection and upload and management of a variety of environmental data.

\section{Data Availability}

The data could be accessed by this link http://miboy.club/ media/data/weather.xlsx.

\section{Conflicts of Interest}

The authors declare that they have no conflicts of interest.

\section{Acknowledgments}

This work was supported in part by the State Key Program of National Natural Science Foundation of China under Grant nos. 61802162 and 41901297, the Science and Technology Key Project of Henan Province under Grant nos. 202102210370 and 202102310017, the Key Research Projects for the Universities of Henan Province (no. 20A170013), and the Science and Technology Foundation of Guizhou Province under Grant no. (2019)1447.

\section{References}

[1] T. Tosco and R. Sethi, "Human health risk assessment for nanoparticle-contaminated aquifer systems," Environmental Pollution, vol. 239, pp. 242-252, 2018.

[2] NASA's Jet Propulsion Laboratory, The Effects of Climate Change, NASA's Jet Propulsion Laboratory, La Cañada Flintridge, CA, USA, 2019, https://climate.nasa.gov/effects/.

[3] European Commission, Causes of Climate Change, European Commission, Brussels, Belgium, 2019, https://ec.europa.eu/ clima/change/causes_en.

[4] P. S. Kench, R. F. McLean, S. D. Owen et al., "Climate-forced sea-level lowstands in the Indian Ocean during the last two millennia," Nature Geoscience, vol. 13, no. 1, pp. 61-64, 2020.

[5] P. Masek, J. Masek, P. Frantik et al., "A harmonized perspective on transportation management in smart cities: the novel IoT-driven environment for road traffic modeling," Sensors, vol. 16, no. 11, p. 1872, 2016.

[6] K. B. Shaban, A. Kadri, and E. Rezk, "Urban air pollution monitoring system with forecasting models," IEEE Sensors Journal, vol. 16, no. 8, pp. 2598-2606, 2016.

[7] D. Balageas, C. P. Fritzen, and A. Güemes, Structural Health Monitoring, John Wiley \& Sons, Hoboken, NJ, USA, 2010.

[8] J. Segura-Garcia, S. Felici-Castell, J. J. Perez-Solano, M. Cobos, and J. M. Navarro, "Low-cost alternatives for urban noise nuisance monitoring using wireless sensor networks," IEEE Sensors Journal, vol. 15, no. 2, pp. 836-844, 2014.

[9] M. A. Hannan, M. Arebey, R. A. Begum, and H. Basri, "Radio Frequency Identification (RFID) and communication technologies for solid waste bin and truck monitoring system," Waste Management, vol. 31, no. 12, pp. 2406-2413, 2011.
[10] M. Srbinovska, C. Gavrovski, V. Dimcev, A. Krkoleva, and V. Borozan, "Environmental parameters monitoring in precision agriculture using wireless sensor networks," Journal of Cleaner Production, vol. 88, pp. 297-307, 2015.

[11] X. Zhang, J. Du, C. Fan, D. Liu, J. Fang, and L. Wang, "A wireless sensor monitoring node based on automatic tracking solar-powered panel for paddy field environment," IEEE Internet of Things Journal, vol. 4, no. 5, pp. 1304-1311, 2017.

[12] N. A. A. Ali and N. A. A. Latiff, "Environmental monitoring system based on LoRa technology in island," in Proceedings of the 2019 IEEE International Conference on Signals and Systems (ICSigSys), pp. 160-166, Bandung, Indonesia, July 2019.

[13] R. K. Saini, "Monitoring water quality by sensors in wireless sensor networks-a review," IITM Journal of Management and IT, vol. 10, no. 1, pp. 1-5, 2019.

[14] H. D. Ahlawat and R. P. Chauhan, "Detection and monitoring of forest fire using serial communication and $\mathrm{Wi}$-Fi wireless sensor network," in Handbook of Wireless Sensor Networks: Issues and Challenges in Current Scenario's, pp. 464-492, Springer, Berlin, Germany, 2020.

[15] B. Potter, G. Valentino, L. Yates, T. Benzing, and A. Salman, "Environmental monitoring using a drone-enabled wireless sensor network," in Proceedings of the 2019 Systems and Information Engineering Design Symposium (SIEDS), pp. 1-6, Charlottesville, VA, USA, April 2019.

[16] M. Carminati, O. Kanoun, S. L. Ullo, and S. Marcuccio, "Prospects of distributed wireless sensor networks for urban environmental monitoring," IEEE Aerospace and Electronic Systems Magazine, vol. 34, no. 6, pp. 44-52, 2019.

[17] V. B. Patil, “ARM based environmental monitoring system for IOT applications," Journal of the Gujarat Research Society, vol. 21, no. 16, pp. 1442-1452, 2019.

[18] L. Joris, F. Dupont, P. Laurent, P. Bellier, S. Stoukatch, and J.-M. Redouté, "An autonomous sigfox wireless sensor node for environmental monitoring," IEEE Sensors Letters, vol. 3, no. 7, pp. 1-4, 2019.

[19] S. Maitra, A. Abdelgawad, and K. Yelamarthi, "Lab in a box: a rapidly deployable environmental monitoring IoT system," in Proceedings of the 2019 IEEE 62nd International Midwest Symposium on Circuits and Systems (MWSCAS), pp. 472-475, Dallas, TX, USA, August 2019.

[20] Ž. Korošak, N. Suhadolnik, and A. Pleteršek, "The implementation of a low power environmental monitoring and soil moisture measurement system based on UHF RFID," Sensors, vol. 19, no. 24, p. 5527, 2019.

[21] S. Amendola, R. Lodato, S. Manzari, C. Occhiuzzi, and G. Marrocco, "RFID technology for IoT-based personal healthcare in smart spaces," IEEE Internet of Things Journal, vol. 1, no. 2, pp. 144-152, 2014.

[22] G. Mois, S. Folea, and T. Sanislav, "Analysis of three IoTbased wireless sensors for environmental monitoring," IEEE Transactions on Instrumentation and Measurement, vol. 66, no. 8, pp. 2056-2064, 2017.

[23] M. N. Al Azam, I. Arif, D. Rizaludin, U. Ayuba, A. Haris, and A. Nugroho, "Wireless sensor network using wireless fidelity for environmental monitoring system," in Proceedings of the 2019 International Seminar on Application for Technology of Information and Communication (iSemantic), pp. 1-7, Semarang, Indonesia, October 2019.

[24] T. M. Behera, S. K. Mohapatra, U. C. Samal, M. S. Khan, M. Daneshmand, and A. H. Gandomi, "I-SEP: an improved routing protocol for heterogeneous WSN for IoT based environmental monitoring," IEEE Internet Things Journal, vol. 7, no. 1, pp. 710-717, 2019. 
[25] A. Ahmad, M. A. Pasha, S. Masud, M. Schappacher, A. Belhouichet, and A. Sikora, "Multi-hop routing implementation in spatially distributed wireless sensor networks for large-scale environmental monitoring applications," in Proceedings of the 2019 10th IEEE International Conference on Intelligent Data Acquisition and Advanced Computing Systems: Technology and Applications (IDAACS), pp. 111-116, Metz, France, September 2019.

[26] M. Wu, L. Tan, and N. Xiong, "Data prediction, compression, and recovery in clustered wireless sensor networks for environmental monitoring applications," Information Sciences, vol. 329, pp. 800-818, 2016.

[27] S. Bezzateev, N. Voloshina, K. Zhidanov, and A. Ometov, "Secure environmental monitoring for industrial internet of things: from framework to live implementation," in Proceedings of the 9th International Conference on Localization and GNSS (ICL-GNSS), pp. 1-5, Nuremberg, Germany, June 2019.

[28] X. Zheng and S. Hou, "Design of fault concentrator based on STM32 and $\mu$ C/OS-II," in Proceedings of the 2016 2nd IEEE International Conference on Computer and Communications (ICCC), pp. 2673-2676, Chengdu, China, October 2016. 